\title{
ARC-SMOOTH CONTINUA
}

\author{
BY
}

\author{
J. B. FUGATE, G. R. GORDH, JR. AND LEWIS LUM
}

\begin{abstract}
ABSTracr. Continua admitting arc-structures and arc-smooth continua are introduced as higher dimensional analogues of dendroids and smooth dendroids, respectively. These continua include such spaces as: cones over compacta, convex continua in $l_{2}$, strongly convex metric continua, injectively metrizable continua, as well as various topological semigroups, partially ordered spaces, and hyperspaces. The arc-smooth continua are shown to coincide with the freely contractible continua and with the metric $K$-spaces of Stadtlander. Known characterizations of smoothness in dendroids involving closed partial orders, the set function $T$, radially convex metrics, continuous selections, and order preserving mappings are extended to the setting of continua with arc-structures. Various consequences of the special contractibility properties of arc-smooth continua are also obtained.
\end{abstract}

Introduction. The purpose of this paper is to introduce and study a well-behaved class of arc-wise connected metric continua called arc-smooth continua. ${ }^{1}$ The class of arc-smooth continua, which may be considered as a higher dimensional analogue of the smooth dendroids [8], includes (a) smooth dendroids; (b) convex (more generally star-like) continua in $l_{2}$; (c) cones over compacta; (d) strongly convex metric continua; (e) injectively metrizable continua [17]; (f) continua ruled by arcs [22]; (g) continuum semilattices with unit; (h) various hyperspaces of continua; and (i) certain partially ordered spaces. The arc-smooth continua coincide with the (a) freely contractible continua [17]; (b) metric $K$-spaces [36]; and (c) ruled metric spaces [9].

Our study of these continua involves a new concept called an arc-structure which is patterned after the "natural arc-structure" possessed by dendroids. In order to motivate the definition, we begin by considering the special case of dendroids.

Let $X$ be a dendroid (i.e., an arc-wise connected, hereditarily unicoherent metric continuum). Each pair of points $x \neq y$ in $X$ determines a unique arc from $x$ to $y$ denoted by $x y$. By convention, $x x=\{x\}$. Denote by $C(X)$ the hyperspace of subcontinua of $X$ with the Hausdorff metric (see [19]). The function $A: X \times X \rightarrow$ $C(X)$ defined by $A(x, y)=x y$ satisfies the following metric-like axioms for all $x, y$ and $z$ in $X$.

(a) $A(x, x)=\{x\}$

Received by the editors July 10, 1979 and, in revised form, May 7, 1980.

AMS (MOS) subject classifications (1970). Primary 54F20; Secondary 54F05.

Key words and phrases. Continuous selection, contractibility, convex metric, dendrite, dendroid, fixed point set, hyperspace of subcontinua, order preserving mapping, partially ordered space, set function $T$, smooth continuum, smooth dendroid, thread action, topological semigroup.

${ }^{1}$ The concept of arc-smooth continua and some of the results in this paper were announced in [12]. The reader is referred to [12] for a discussion of the relationship of arc-smooth continua to several other generalizations of smooth dendroids which have been studied in recent years. 
(b) $A(x, y)=A(y, x)$, and

(c) $A(x, z) \subseteq A(x, y) \cup A(y, z)$

with equality prevailing whenever $y$ belongs to $A(x, z)$.

Furthermore, it is easy to see that the dendroid $X$ is smooth [8] at the point $p$ precisely when the induced function $A_{p}: X \rightarrow C(X)$ defined by $A_{p}(x)=A(p, x)$ is continuous.

We define an arc-structure on an arbitrary continuum $X$ to be a function $A$ : $X \times X \rightarrow C(X)$ such that for $x \neq y$ in $X$, the set $A(x, y)$ is an arc from $x$ to $y$ and such that conditions (a)-(c) are satisfied. The pair $(X, A)$ is called arc-smooth at the point $p$ in $X$ provided the induced function $A_{p}: X \rightarrow C(X)$ is continuous. The pair $(X, A)$ is arc-smooth in case there exists a point in $X$ at which $(X, A)$ is arc-smooth.

An arbitrary continuum $X$ is said to be arc-smooth at the point $p$ provided there exists an arc-structure $A$ on $X$ for which $(X, A)$ is arc-smooth at $p$. The continuum $X$ is arc-smooth if it is arc-smooth at some point. We shall observe in §II that this definition is equivalent to the definition originally given in [12].

The paper is divided into two major sections. In $\S I$ we study pairs $(X, A)$ where $X$ is a continuum with a fixed arc-structure $A$, and we consider two general questions: (1) What properties must $X$ and $A$ possess if $(X, A)$ is arc-smooth? (2) What properties of $X$ and $A$ imply that $(X, A)$ is arc-smooth? Many of the results generalize known theorems concerning smoothness in dendroids. In §II we study analogous questions for arbitrary continua: (1) What properties must arc-smooth continua possess? (2) What properties imply that a continuum is arc-smooth?

Preliminaries. By a continuum we mean a compact connected metric space. The continuum $X$ is unicoherent provided the intersection of any two subcontinua whose union is $X$ is connected; and $X$ is hereditarily unicoherent in case each subcontinuum is unicoherent. A dendroid is an arcwise connected hereditarily unicoherent continuum. A dendrite is a locally connected dendroid.

If $X$ is a continuum, then $C(X)$ denotes the hyperspace of subcontinua of $X$ with the Hausdorff metric. The reader is referred to [19] and [33] for basic definitions and facts concerning $C(X)$.

If $Z$ is a subset of the continuum $X$, then $\mathrm{cl} Z$ will denote the closure of $Z$ and int $Z$ will denote the interior of $Z$. If $\left\{Z_{n}\right\}$ is a sequence of subsets of $X$, then Li $Z_{n}$, Ls $Z_{n}$, and $\operatorname{Lim} Z_{n}$ will denote the limit inferior, the limit superior, and the limit of $\left\{Z_{n}\right\}$, respectively.

\section{Arc-smoothness for continua with arc-structures.}

Convention. Throughout $\S \mathrm{I}, X$ denotes a continuum with a given arc-structure $A$ : $X \times X \rightarrow C(X)$ as defined in the Introduction. Given $x$ and $y$ in $X$, the $\operatorname{arc} A(x, y)$ will be denoted by $x y$.

Recall that the pair $(X, A)$ is said to be arc-smooth at $p$ provided the induced function $A_{p}: X \rightarrow C(X)$ is continuous.

I.1. The order $\leqslant_{p}$. For each $p$ in $X$ we define the partial order $<_{p}$ by letting $x \leqslant_{p} y$ whenever $p x \subseteq p y$. When $X$ is a dendroid, $\leqslant_{p}$ is simply the familiar weak cutpoint order with respect to $p$ (see [21]). 
For a subset $H$ of $X$ the lower set $L_{p}(H)$ is the set $\left\{x \in X \mid x<_{p} y\right.$ for some $y \in H\}$; and the upper set $M_{p}(H)$ is the set $\left\{y \in X \mid x<_{p} y\right.$ for some $\left.x \in H\right\}$.

THEOREM I-1-A. The following are equivalent.

(a) $(X, A)$ is arc-smooth at $p$.

(b) $\leqslant_{p}$ is closed in $X \times X$.

(c) For each closed subset $H$, the upper set $M_{p}(H)$ is closed.

(d) For each closed subset $H$, the lower set $L_{p}(H)$ is closed; and the upper set $M_{p}(x)$ is closed for each $x$ in $X$.

(e) Whenever $x$ belongs to an open set $W$ such that $W=M_{p}(W)$ there exists an open set $U$ such that $U=M_{p}(U)$ and $x \in U \subseteq \operatorname{cl} U \subseteq W$.

(Observe that (e) is a kind of regularity condition for open upper sets.)

Proof. We shall show that (b) is equivalent to each of the other conditions.

(a) implies (b). Assume that $\left\{x_{n}\right\}$ and $\left\{y_{n}\right\}$ are sequences converging to $x$ and $y$, respectively, and that $x_{n} \leqslant p y_{n}$ for each $n$. By arc-smoothness, $\left\{p y_{n}\right\}$ converges to py. Since $x_{n} \in p y_{n}$, it follows that $x \in p y$. Thus $x<_{p} y$ as required.

(b) implies (a). To prove that $A_{p}: X \rightarrow C(X)$ is continuous, it suffices to show that if $\left\{y_{n}\right\}$ converges to $y$ in $X$, then $\left\{p y_{n}\right\}$ converges to $p y$ in $C(X)$. Let $x \in \operatorname{Ls} p y_{n}$. There is a subsequence $\left\{p y_{n_{i}}\right\}$ such that each $p y_{n_{i}}$ contains a point $x_{n_{i}}$ so that $\left\{x_{n_{i}}\right\}$ converges to $x$. Since $<_{p}$ is closed, $x \leqslant_{p} y$. Consequently Ls $p y_{n} \subseteq p y$. But Ls $p y_{n}$ is a continuum (Theorem 2-101 of [16]) containing $p$ and $y$; hence Ls $p y_{n}=p y$. Furthermore, Li $p y_{n}=p y$. For if $x \in p y \backslash \mathrm{Li} p y_{n}$, there is an open set $U$ containing $x$ and a subsequence $\left\{y_{n_{j}}\right\}$ of $\left\{y_{n}\right\}$ such that $U \cap p y_{n_{j}}=\varnothing$ for all $j$. It follows that Ls $p y_{n_{j}}$ is a subcontinuum of $p y$ which contains $p$ and $y$ but not $x$. This contradiction shows that $\mathrm{Ls} p y_{n}=\mathrm{Li} p y_{n}=p y$. Thus $\left\{p y_{n}\right\}$ converges to $p y$.

(c) implies (b). To see that $\leqslant_{p}$ is closed, suppose that $x \leqslant_{p} y$. Choose an open set $U$ such that $x \in U \subseteq \operatorname{cl} U \subseteq X \backslash p y$. Thus $M_{p}(\operatorname{cl} U)$ is closed and $p y \cap$ $M_{p}(\mathrm{cl} U)=\varnothing$. Let $V$ be an open set such that $y \in V \subseteq X \backslash M_{p}(\mathrm{cl} U)$. Now if $(s, t) \in U \times V$, then $s \$ p_{p} t$ (otherwise $t \in M_{p}(\mathrm{cl} U) \cap V=\varnothing$ ).

(b) implies (c). Obvious.

(d) implies (b). To prove that $\leqslant_{p}$ is closed, suppose that $x \nless_{p} y$. Since $M_{p}(x)$ is closed there is an open set $V$ such that $y \in V \subseteq \operatorname{cl} V \subseteq X \backslash M_{p}(x)$. Now $L_{p}(\mathrm{cl} V)$ is a closed set which misses $M_{p}(x)$. There is an open set $U$ such that $M_{p}(x) \subseteq U \subseteq$ $X \backslash L_{p}(\mathrm{cl} V)$. Now if $(s, t) \in U \times V$, then $s \Varangle_{p} t$ as required.

(b) implies (d). Obvious.

(e) implies (b). Suppose that $x \$_{p} y$. Then $W=X \backslash L_{p}(y)$ is an open set containing $x$ such that $W=M_{p}(W)$. By hypothesis there is an open set $U$ such that $U=M_{p}(U)$ and $x \in U \subseteq \operatorname{cl} U \subseteq W$. Let $V$ be an open set such that $y \in V \subseteq$ $X \backslash \mathrm{cl} U$. It follows that $s \$_{p} t$ whenever $(s, t) \in U \times V$.

(b) implies (e). Let $W$ be an open set containing $x$ such that $W=M_{p}(W)$. Let $V$ be an open set such that $x \in V \subseteq \operatorname{cl} V \subseteq W$ and let $U=M_{p}(V)$. To see that $U$ is open let $y \in U$ and let $\left\{y_{n}\right\}$ be a sequence converging to $y$. Since $y \in M_{p}(V)$, the $\operatorname{arc} p y$ intersects $V$. Thus, by (a), for sufficiently large $n$, the $\operatorname{arc} p y_{n}$ intersects $V$ and hence $y_{n} \in U$. A similar argument shows that cl $U \subseteq W$. 
REMARK. Some related results for uniquely arcwise connected continua appear in [35].

I.2. Convex sets. A subset $Z$ of $X$ is said to be convex if for each pair of points $x$ and $y$ of $Z$ the arc $x y$ is a subset of $Z$. Observe that if $Z$ is a convex subcontinuum of $X$, then $A \mid Z \times Z$ is an arc-structure on $Z$.

THEOREM I-2-A. (a) The intersection of any collection of convex sets is convex. (b) For each $p \in X$ and subset $H$ the lower set $L_{p}(H)$ is convex. (c) For each $p \in X$ and convex subset $Z$ the upper set $M_{p}(Z)$ is convex. (d) If $Z$ is a convex subcontinuum of $X$, then for each $p \in X$ the set $Z$ has a zero relative to $\leqslant_{p}$.

Proof. (a), (b) and (c) follow immediately from the definition. To prove (d) let $x$ be an arbitrary point in $Z$ and let $z$ be the first point on the $\operatorname{arc} p x$ which belongs to $Z$. If $z \nless p_{p} y$ for some $y \in Z$, then $z \notin p y$ and $p z \cap p y \subseteq X \backslash Z$. Since $z y \subseteq p z$ $\cup$ py it follows that $z y$ is not contained in $Z$ which contradicts the assumption that $Z$ is convex.

Lemma I-2-B. Suppose that $(X, A)$ is arc-smooth at $p$.

(a) If $\left\{Z_{n}\right\}$ is a sequence of convex subsets of $X$ and $\operatorname{Li} Z_{n} \neq \varnothing$, then $\operatorname{Ls} Z_{n}$ and Li $Z_{n}$ are convex.

(b) If $\left\{x_{n}\right\}$ and $\left\{y_{n}\right\}$ are sequences converging to $x$ and $y$, respectively, and $x_{n} \leqslant p y_{n}$ for every $n$, then the sequence of arcs $\left\{x_{n} y_{n}\right\}$ converges to the arc $x y$.

(c) If $Z$ is a convex subcontinuum of $X$ and $z$ is the zero of $Z$ relative to $\leqslant_{p}$, then $(Z, A \mid Z \times Z)$ is arc-smooth at $Z$.

(d) If $Z$ is a convex subset of $X$, then $\mathrm{cl} Z$ is convex.

Proof. (a) To show that Ls $Z_{n}$ is convex it suffices to prove that $x y \subseteq$ Ls $Z_{n}$ whenever $x \in \operatorname{Li} Z_{n}$ and $y \in \operatorname{Ls} Z_{n}$. Passing to subsequences if necessary, we may assume that there are sequences $\left\{x_{n}\right\}$ and $\left\{y_{n}\right\}$ converging to $x$ and $y$, respectively, with $x_{n}$ and $y_{n}$ belonging to $Z_{n}$ for each $n$. Notice that for each $n$ the arc $x_{n} y_{n}$ is contained in $Z_{n} \cap\left(p x_{n} \cup p y_{n}\right)$. By arc-smoothness, $\operatorname{Ls}\left(p x_{n} \cup p y_{n}\right)=p x \cup p y$. Thus Ls $x_{n} y_{n}$ is a subcontinuum of $\left(\operatorname{Ls} Z_{n}\right) \cap(p x \cup p y)$ which contains $x$ and $y$. It follows that $x y \subseteq \operatorname{Ls} x_{n} y_{n} \subseteq$ Ls $Z_{n}$ as required.

Suppose that $\mathrm{Li} Z_{n}$ is not convex and let $x$ and $y$ be points of $\mathrm{Li} Z_{n}$ for which $x y \nsubseteq \operatorname{Li} Z_{n}$. Let $z \in x y \backslash \operatorname{Li} Z_{n}$ and let $U$ be an open set such that $z \in U \subseteq$ $X \backslash \operatorname{Li~} Z_{n}$. There exists a subsequence $\left\{Z_{n_{i}}\right\}$ of $\left\{Z_{n}\right\}$ and points $x_{n_{i}}, y_{n_{i}} \in Z_{n_{i}}$ such that $Z_{n_{i}} \cap U=\varnothing$ for each $i,\left\{x_{n_{i}}\right\}$ converges to $x$, and $\left\{y_{n_{i}}\right\}$ converges to $y$. Thus $\operatorname{Ls}\left(p x_{n_{i}} \cup p y_{n_{i}}\right) \cap U=\varnothing$. By arc-smoothness, $x y \subseteq p x \cup p y=\operatorname{Ls}\left(p x_{n_{i}} \cup p y_{n_{i}}\right)$. Thus $x y \cap U=\varnothing$ contradicting the choice of $z$.

(b) By (a) $x y \subseteq \operatorname{Li} x_{n} y_{n}$; hence it suffices to show that Ls $x_{n} y_{n} \subseteq x y$. Let $z \in \operatorname{Ls} x_{n} y_{n}$. Without loss of generality there is a sequence $\left\{z_{n}\right\}$ converging to $z$ with $z_{n} \in x_{n} y_{n}$. Thus $x_{n} \leqslant p z_{n} \leqslant p y_{n}$, and, by Theorem I-1-A, $x<_{p} z<_{p} y$. Consequently $z \in x y$.

(c) Given $\left\{y_{n}\right\}$ in $Z$ converging to $y$, apply (b) to see that $\operatorname{Lim} z y_{n}=z y$.

(d) Let $Z_{n}=Z$ for each $n$. Then, by (a), cl $Z=$ Ls $Z_{n}$ is convex. 
We define $X$ to be locally convex at a subcontinuum $M$ if for each open set $U$ containing $M$, there is a convex set $Z$ such that $M \subseteq$ int $Z \subseteq \operatorname{cl} Z \subseteq U$. In case $M$ consists of a single point $p$ we say that $X$ is locally convex at $p$.

If $Y$ is a subset of $X$, then by a convex component of $Y$ we mean a maximal convex subset of $Y$.

THEOREM I-2-C. The following are equivalent.

(a) $(X, A)$ is arc-smooth at $p$.

(b) For each open set $U$ containing $p$, the convex component of $U$ which contains $p$ is open.

(c) $X$ is locally convex at each convex subcontinuum which contains $p$.

Proof. (a) implies (b). If $Z$ denotes the convex component of $U$ which contains $p$, then $Z=\{x \in U \mid p x \subseteq U\}$. If $Z$ is not open, then for some $x \in Z$ there exists a sequence $\left\{x_{n}\right\}$ in $X \backslash Z$ which converges to $x$. Then for each $n$, the set $p x_{n} \cap$ $(X \backslash U)$ is nonempty. Consequently $\left(\operatorname{Ls} p x_{n}\right) \cap(X \backslash U) \neq \varnothing$. By arc-smoothness $p x \cap(X \backslash U) \neq \varnothing$ which contradicts the fact that $p x \subseteq U$.

(b) implies (c). Let $p \in M \subseteq U$ where $M$ is a convex subcontinuum and $U$ is an open set. Let $V$ be an open set such that $M \subseteq V \subseteq$ cl $V \subseteq U$. If $Z$ denotes the convex component of $V$ which contains $p$, then $Z$ is open by (b), and, furthermore, $M \subseteq$ int $Z=Z \subseteq$ cl $Z \subseteq U$ as needed.

(c) implies (a). By Theorem I-1-A, it suffices to show that $<_{p}$ is closed. Suppose that $\left\{x_{n}\right\}$ and $\left\{y_{n}\right\}$ are sequences with $x_{n} \leqslant p y_{n}$ for each $n$ which converge to $x$ and $y$, respectively. If $x \$_{p} y$, then there exists an open set $U$ which contains $p y$ and misses $x$. By (c) there is a convex set $Z$ such that $p y \subseteq$ int $Z \subseteq \operatorname{cl} Z \subseteq U$. This contradicts the fact that for sufficiently large $n$, the arc $p y_{n}$ lies in $Z$.

REMARK. An analogous result is valid for continua which are smooth in the sense of Mackowiak [31, Theorem (3.1)].

Corollary I-2-D. If $(X, A)$ is arc-smooth at $p$, then $X$ is locally convex at $p$.

THEOREM I-2-E. The following are equivalent.

(a) $(X, A)$ is arc-smooth at each point.

(b) $X$ is locally convex at each point.

(c) $X$ is a dendrite.

Proof. (a) implies (b). Corollary I-2-D.

(b) implies (c). It suffices to show that each pair of distinct points $x$ and $y$ can be separated by a third point (see [42, p. 88]). Let $z \in x y \backslash\{x, y\}$ and observe that $x$ and $y$ lie in distinct convex components of $X \backslash\{z\}$. Moreover, every convex component of $X \backslash\{z\}$ is open by (b). Thus $z$ separates $x$ from $y$ in $X$.

(c) implies (a). Dendrites are smooth (hence arc-smooth) at each point by Corollary 4 of [8].

Notation. Let $I(X)=\{p \in X \mid(X, A)$ is arc-smooth at $p\} . I(X)$ is called the initial set of $X$. Let $N(X)=\{x \in X \mid X$ is not locally convex at $x\}$. 
The sets $I(X)$ and $N(X)$ were introduced in [8] for dendroids. Our next result generalizes Theorem 2 of [8]. Since the proof carries over with only minor modifications, it will be omitted.

TheOREM I-2-F. If $(X, A)$ is arc-smooth at $p$, then the initial set $I(X)$ is the convex component of $X \backslash N(X)$ which contains $p$.

I.3. A generalization of the set function $T$. Jones [18] defined the set function $T$ (which he called $L$ ) on any continuum $M$ by the equation $T(x)=\{y \in M \mid$ each subcontinuum of $M$ with $y$ in its interior contains $x\}$. This set function has been used to characterize smoothness in dendroids [8] and hereditarily unicoherent continua [13].

Here we modify $T$ so that it is applicable to continua with arc-structures and use it to characterize arc-smoothness.

For each point $x$ of the continuum $X$ with arc-structure $A$ we define $T_{A}(x)$ to be the set $\{y \in X \mid$ each convex subcontinuum of $X$ with $y$ in its interior contains $x\}$.

It is clear from the definitions that (a) $T(x) \subseteq T_{A}(x)$ for all $x \in X$, (b) $T(x)$ and $T_{A}(x)$ are always closed, and (c) $T$ and $T_{A}$ agree whenever $X$ is a dendroid. The simple example which follows shows that, unlike $T(x)$, the set $T_{A}(x)$ need not be connected. Observe that $T_{A}(x)$ is connected whenever $(X, A)$ is arc-smooth by Theorem I-3-B.

EXAMPLE. Let $X$ denote the unit circle in the plane, let $p \in X$, and let $\leqslant$ order $X$ in a clockwise manner away from $p$. Define $A: X \times X \rightarrow C(X)$ by the equation $A(x, y)=\{z \in X \mid x \leqslant z \leqslant y\}$. Now $A$ is an arc-structure on $X$, although $(X, A)$ is not arc-smooth at any point. For any $q \neq p$, we have $T_{A}(q)=\{p, q\}$.

TheOREM I-3-A. If $(X, A)$ is arc-smooth at $p$, then $T_{A}(x) \subseteq M_{p}(x)$ for all $x \in X$.

Proof. Suppose $y \notin M_{p}(x)$. Then $X \backslash M_{p}(x)$ is an open set which contains $p y$ by Theorem I-1-A. By Theorem I-2-C, $X$ is locally convex at $p y$. It follows that $y \notin T_{A}(x)$.

THEOREM I-3-B. $(X, A)$ is arc-smooth at $p$ if and only if for each $x$ in $X$ the following hold.

(a) $T_{A}(x)$ is convex, and

(b) $p x \cap T_{A}(x)=\{x\}$.

Proof. Suppose that $(X, A)$ is arc-smooth at $p$. Then (b) follows immediately from Theorem I-3-A. To prove (a), let $y \in T_{A}(x)$ and note that $x<p y$. If $x y \nsubseteq T_{A}(x)$ then there is a point $z \in x y$ and a convex continuum $Z$ with $z \in$ int $Z$ $\subseteq Z \subseteq X \backslash\{x\}$. Observe that $x \leqslant_{p} w$ for each $w \in Z$ (otherwise $x \notin p w$ and hence $x \notin p z \subseteq p w \cup w z)$. Now $M_{p}(Z)$ is a convex subcontinuum of $X$ with $y$ in its interior which misses $x$. This contradicts $y \in T_{A}(x)$. Thus $x y \subseteq T_{A}(x)$ and $T_{A}(x)$ is convex.

Suppose that (a) and (b) hold. It suffices by Theorem I-1-A to show that $<_{p}$ is closed. Suppose $x \$_{p} y$ and observe that (a) and (b) imply that $y \notin T_{A}(x)$. Thus there is a convex subcontinuum $Z$ such that $y \in$ int $Z \subseteq Z \subseteq X \backslash\{x\}$. Thus 
py $\cup Z$ is a convex continuum missing $x$. Now if $(s, t) \in[X \backslash(p y \cup Z)] \times$ int $Z$, then $s \notin p t \subseteq p y \cup Z$. Hence $s \$_{p} t$ and $\leqslant_{p}$ is closed.

TheOREM I-3-C. $X$ is a dendrite if and only if $T_{A}(x)=\{x\}$ for all $x$ in $X$.

Proof. If $X$ is a dendrite, then $T_{A}(x)=T(x)=\{x\}$ for each $x$.

If $T_{A}(x)=\{x\}$ for each $x$, then (a) and (b) of Theorem I-3-B are satisfied for each $p$ in $X$. Consequently, $X$ is arc-smooth at each point, and, by Theorem I-2-E, $X$ is a dendrite. (Alternately, one can easily establish local convexity at each point.)

THEOREM I-3-D. $(X, A)$ is arc-smooth if and only if for each $x$ and $y$ in $X$ the following hold.

(a) $T_{A}(x)$ is convex, and

(b) $x y \cap T_{A}(x)=\{x\}$ or $x y \cap T_{A}(y)=\{y\}$.

PROof. The proof parallels the one for dendroids (Theorem 6 of [8]) provided it is shown that conditions (a) and (b) imply that if $\left\{x_{n}\right\}$ is a sequence of points converging to $x$ such that $x_{n} \leqslant p x_{n+1}$ for every $n$, then $\operatorname{cl}\left(\cup p x_{n}\right)=p x$. Observe that it suffices to show that $x_{n} \in p x$ for each $n$. Suppose this is not the case, and without loss of generality assume that $x_{n} \notin p x$ for all $n$. Let $y=\sup \left(p x_{1} \cap p x\right)$ and observe that $y \in x x_{n}$ for all $n$. Suppose that $Z$ is a convex subcontinuum containing $x$ in its interior. Then, for sufficiently large $n$, it follows that $x x_{n} \subseteq Z$, hence $y \in Z$ and $x_{n} \in Z$ for all $n$. Consequently, $x \in T_{A}(y)$ and $x \in T_{A}\left(x_{n}\right)$ for all $n$. By (a), $T_{A}\left(x_{n}\right)$ is convex, and hence $y \in T_{A}\left(x_{n}\right)$ for all $n$. It follows that $y \in T_{A}(x)$. Thus $x \in T_{A}(y)$ and $y \in T_{A}(x)$, contradicting (b).

I.4. Radially convex metrics. A metric $d$ on $X$ is called radially convex at the point $p$ provided that $d(p, z)=d(p, y)+d(y, z)$ whenever $y \in p z$. Observe that this implies that each arc $p z$ is congruent to a line segment. Also, for each $\varepsilon>0$, the set $N_{\varepsilon}(p)=\{x \in X \mid d(p, x) \leqslant \varepsilon\}$ is a convex subcontinuum of $X$.

THEOREM I-4-A. $(X, A)$ is arc-smooth at $p$ if and only if the following hold.

(a) $T_{A}(x)$ is convex for each $x$ in $X$, and

(b) $X$ admits a metric $d$ which is radially convex at $p$.

Proof. Assume that $(X, A)$ is arc-smooth at $p$. Theorem I-3-B establishes (a). By Theorem I-1-A, $\leqslant_{p}$ is a closed partial order. Let $H$ denote the Hilbert cube with the coordinatewise partial order, and let the distance between points $\left\{x_{n}\right\}$ and $\left\{y_{n}\right\}$ in $H$ be given by $\Sigma\left|x_{n}-y_{n}\right| / 2^{n}$. Carruth has shown (Theorem 1 of [6] or Corollary 1.5 of [5]) that there is an order preserving embedding of $X$ into $H$. The induced metric $d$ on $X$ is easily seen to be radially convex at $p$.

Now suppose that (a) and (b) hold. By Theorem I-3-B, it suffices to show that $p x \cap T_{A}(x)=\{x\}$ for each $x \in X$. Suppose that $y \in p x \backslash\{x\}$, and let $\varepsilon$ be a real number such that $d(p, y)<\varepsilon<d(p, x)$. Then $N_{\varepsilon}(p)$ is a convex subcontinuum containing $y$ in its interior and missing $x$. Hence $y \notin T_{A}(x)$ as required.

THEOREM I-4-B. $X$ is a dendrite if and only if for each $p$ in $X$ there exists a metric on $X$ which is radially convex at $p$. 
Proof. By Theorem I-2-E, it suffices to note that the existence of a metric on $X$ which is radially convex at $p$ implies that $X$ is locally convex at $p$ (consider $N_{e}(p)$ ).

I.5. Continuous selections. Let Convex $(X)$ denote the subspace of $C(X)$ consisting of all convex subcontinua of $X$.

For each $p$ in $X$ we define a selection $\sigma_{p}$ : Convex $(X) \rightarrow X$ by letting $\sigma_{p}(Z)$ be the unique zero of $Z$ relative to $\leqslant_{p}$ (see Theorem I-2-A).

Observe that $X$ is a dendroid precisely when Convex $(X)=C(X)$. In this case, $\sigma_{p}$ is the so-called least element function. Our next theorem generalizes a result of Ward [40]: the dendroid $X$ is smooth at $p$ if and only if the least element function $\sigma_{p}$ is continuous.

THEOREM I-5-A. $(X, A)$ is arc-smooth at $p$ if and only if the following hold.

(a) Convex $(X)$ is closed in $C(X)$, and

(b) $\sigma_{p}:$ Convex $(X) \rightarrow X$ is continuous.

Proof. Assume that $(X, A)$ is arc-smooth at $p$. Lemma I-2-B implies (a). Condition (b) follows easily from the definition of $\sigma_{p}$ and the fact that $<_{p}$ is closed.

Suppose (a) and (b) hold. To show that $<_{p}$ is closed, let $\left\{x_{n}\right\}$ and $\left\{y_{n}\right\}$ be sequences in $X$ converging to $x$ and $y$, respectively, such that $x_{n}<_{p} y_{n}$ for each $n$. Then $\left\{x_{n} y_{n}\right\}$ is a sequence in $\operatorname{Convex}(X)$ which, without loss of generality, converges to a convex subcontinuum $Z$. By $(b), \sigma_{p}(Z)=x$. Hence $x<_{p} y$ and $<_{p}$ is closed.

RemarK. The example in (I.3) shows that Theorem I-5-A fails without the assumption that Convex $(X)$ be closed in $C(X)$.

I.6. $<_{p}$-contractibility. Given $p$ in $X$, we define a $<_{p}$-contraction to be a homotopy $H: X \times[0,1] \rightarrow X$ satisfying the following conditions for all $x$ in $X$ :

(a) $H(x, 1)=x$,

(b) $H(x, 0)=p$, and

(c) $H(x, t) \leqslant_{p} x$ for all $t \in[0,1]$.

TheOREM I-6-A. $(X, A)$ is arc-smooth at $p$ if and only if $X$ admits $a<_{p}$-contraction.

Proof. Suppose that $(X, A)$ is arc-smooth at $p$ and let $d$ be a metric on $X$ which is radially convex at $p$ and bounded by 1 . Following Mohler's construction of a retracting homotopy for smooth dendroids (Theorem 1.16 of [32]) we define $H$ : $X \times I \rightarrow X$ as follows:

(i) If $d(p, x) \leqslant t$, then $H(x, t)=x$.

(ii) If $d(p, x) \geqslant t$, then $H(x, t)=y$

where $y \in p x$ and $d(p, y)=t$.

Clearly $H$ satisfies (a), (b) and (c). The continuity of $H$ follows just as in [32].

Now suppose that $H: X \times[0,1] \rightarrow X$ is a $<_{p}$-contraction. We shall show that $<_{p}$ is closed. Assume that $\left\{x_{n}\right\}$ and $\left\{y_{n}\right\}$ are sequences in $X$ converging to $x$ and $y$, respectively, and that $x_{n} \leqslant_{p} y_{n}$ for every $n$. The properties of a $<_{p}$-contraction imply that for each $n$ there is a $t_{n} \in[0,1]$ such that $H\left(y_{n}, t_{n}\right)=x_{n}$. Without loss of generality we may assume that $\left\{\left(y_{n}, t_{n}\right)\right\}$ converges, say to $(y, t)$. Then $x=H(y, t)$ by continuity, and $H(y, t) \leqslant_{p} y$ in (c). Consequently $x<_{p} y$ and $<_{p}$ is closed. 
I.7. Order preserving mappings. Order preserving mappings of various kinds have been applied to obtain characterizations of smoothness (e.g., [8], [10], [14], [27], [29], and [30]). Here we introduce several types of order preserving mappings for continua with arc-structures and use them to characterize arc-smoothness.

Convention. Throughout (I.7) $X$ and $Y$ denote continua with fixed arc-structures $A$ and $B$, respectively.

We say that a continuous surjective function $f: X \rightarrow Y$ is a $<_{p}$-mapping in case $x \leqslant_{p} y$ in $X$ implies that $f(x) \leqslant f(p) f(y)$ in $Y$. If, in addition, $Y \subseteq X, B=A \mid Y \times Y$ and $f$ is a retraction, then $f$ is called a $\leqslant_{p}$-retraction. $<_{p}$-mappings are defined in a similar manner.

Observe that if $f: X \rightarrow Y$ is a $\leqslant_{p}$-mapping and $x \leqslant_{p} y$, then $f(x y)=f(x) f(y)$. From this it follows easily that $f$ preserves convex sets and that $f^{-1}$ preserves convex sets containing $f(p)$.

THEOREM I-7-A. If $f: X \rightarrow Y$ is $a \leqslant_{p}$-mapping and $(X, A)$ is arc-smooth at $p$, then $(Y, B)$ is arc-smooth at $f(p)$.

Proof. It suffices to show that $\leqslant_{f(p)}$ is closed. Let $\left\{y_{n}\right\}$ and $\left\{z_{n}\right\}$ be sequences in $Y$ converging to $y$ and $z$, respectively, and such that $y_{n} \leqslant f(p) z_{n}$ for each $n$. Let $\left\{x_{n}\right\}$ be a sequence in $X$ such that $f\left(x_{n}\right)=z_{n}$ for each $n$. Since $f\left(p x_{n}\right)=f(p) f\left(x_{n}\right)=$ $f(p) z_{n}$, there is a point $w_{n} \in p x_{n}$ such that $f\left(w_{n}\right)=y_{n}$ for each $n$. Without loss of generality we may assume that $\left\{\left(w_{n}, x_{n}\right)\right\}$ converges to $(w, x)$ in $X \times X$. Then $w \leqslant_{p} x$ since $(X, A)$ is arc-smooth at $p$. Consequently $y=f(w) \leqslant_{f(p)} f(x)=z$ and $\leqslant f(p)$ is closed.

The cone over the Cantor set is universal in the mapping sense for the class of smooth dendroids (Theorem 11 of [8]). Our next theorem extends this result to arbitrary arc-smooth continua.

THEOREM I-7-B. Let $X$ be the cone over the Cantor set, $A$ the unique arc-structure on $X$, and $p$ the vertex of $X$. Then $(Y, B)$ is arc-smooth at the point $q$ if and only if there exists $a \leqslant_{p}$-mapping $f: X \rightarrow Y$ such that $f(p)=q$.

Proof. If $f$ exists, then $(Y, B)$ is arc-smooth at $q$ by Theorem I-7-A. If $(Y, B)$ is arc-smooth at $q$, then $Y$ admits a metric which is radially convex at $q$ (Theorem I-4-A) and hence the construction of $f$ given in Theorem 11 of [8] for dendroids is valid for arc-smooth continua.

THEOREM I-7-C. The following are equivalent.

(a) $(X, A)$ is arc-smooth at $p$.

(b) There exists $a<_{p}$-mapping $f: X \rightarrow[0,1]$ with $f(p)=0$, and for each $x$ in $X$ the set $T_{A}(x)$ is convex.

(c) For each $x$ in $X$, there exists $a \leqslant_{p}$-retraction $r: X \rightarrow p x$ and $T_{A}(x)$ is convex.

Proof. (a) implies (b). $T_{A}(x)$ is convex by Theorem I-3-B. Let $d$ be a metric on $X$ which is radially convex with respect to $p$ and such that $\sup \{d(p, x) \mid x \in X\}=1$. Then the mapping $f: X \rightarrow[0,1]$ defined by $f(x)=d(p, x)$ is a $<_{p}$-mapping. 
(b) implies (a). By Theorem I-3-B it suffices to show that $p x \cap T_{A}(x)=\{x\}$ for each $x \in X$. Suppose that $y \in p x \backslash\{x\}$, and choose $t \in[0,1]$ such that $f(y)<t<$ $f(x)$. Then $f^{-1}([0, t])$ is a convex subcontinuum of $X$ which contains $y$ in its interior and misses $x$. Consequently, $y \notin T_{A}(x)$ and $p x \cap T_{A}(x)=\{x\}$.

(a) implies (c). $T_{A}(x)$ is convex by Theorem I-3-B. Let $d$ be a metric which is radially convex with respect to $p$ and which is bounded by 1 . Fix $x \in X$, and let $t=d(p, x)$. Let $H$ be the $\leqslant_{p}$-contraction defined in Theorem I-6-A, and let $f=H \mid X \times\{t\}$. Then $f$ retracts $X$ onto $N_{t}(p)$. Now define $g: N_{t}(p) \rightarrow[0, t]$ by $g(y)=d(p, y)$, and define $h:[0, t] \rightarrow p x$ to be the isometry with $h(0)=p$. Then $r=h \circ g \circ f$ is the required retraction.

(c) implies (a). The proof is similar to the proof that (b) implies (a).

REMARK. If $X$ is a dendroid, then the assumption that $T_{A}(x)$ be convex in Theorem I-7-C is superfluous. Thus for the special case of dendroids, the equivalence of (a) and (b) is Corollary 4 of [14], and the equivalence of (a) and (c) is the main result of [29]. The following example shows that the assumption that $T_{A}(x)$ be convex is not superfluous in general.

EXAMPLE. Let $X$ denote the unit circle in the plane and let $p=(0,-1)$. For each $x$ in $X$, if $p \neq x \neq(0,1)$ define $p x$ to be the shorter arc joining $p$ to $x$. For $x=(0,1)$ let $p x$ be the set of points with nonnegative first coordinates. Let $A$ be the unique arc-structure on $X$ compatible with the collection of arcs just defined. Then $(X, A)$ is not arc-smooth at $p$ even though the function $f: X \rightarrow[0,1]$ defined by the equation $f(x)=d(p, x) / 2$ is a $<_{p}$-mapping.

COROLlARY I-7-D. The following are equivalent.

(a) $X$ is a dendrite.

(b) For each $p$ in $X$ there exists $a<_{p}$-mapping $f: X \rightarrow[0,1]$ with $f(p)=0$.

(c) For each arc $x y$ in $X$ there exists $a \leqslant_{x}$-retraction $r: X \rightarrow x y$.

Proof. That (a) implies (b) and (c) follows immediately from Theorem I-7-C. It is easy to verify that (b) and (c) each implies that $T_{A}(x)=\{x\}$ for each $x$ in $X$. Thus the desired conclusion follows from Theorem I-7-C.

I.8. Convex-monotone mappings. Monotone mappings are known to preserve the class of smooth dendroids [8]. In contrast, simple examples show that monotone mappings need not preserve arc-smooth continua. In this section we define a special class of monotone mappings for continua with arc-structures and show that they preserve arc-smoothness.

Convention. In this section $X$ denotes, as usual, a continuum with a fixed arc-structure $A$, and $Y$ denotes an arbitrary continuum.

We say that a mapping $f: X \rightarrow Y$ is convex-monotone provided that $f^{-1}(y)$ is a convex subcontinuum of $X$ for each $y$ in $Y$.

RemarK. Observe that when $X$ is a dendroid, the convex-monotone mappings coincide with the monotone mappings.

We now observe that if $f: X \rightarrow Y$ is a convex-monotone mapping, then $f$ induces a natural arc-structure, say $B$, on $Y$. Given $y_{1}$ and $y_{2}$ in $Y$, let $x_{1} x_{2}$ be an arc in $X$ which is irreducible from $f^{-1}\left(y_{1}\right)$ to $f^{-1}\left(y_{2}\right)$. Using the convexity of $f^{-1}\left(y_{1}\right)$ and 
$f^{-1}\left(y_{2}\right)$ it is easy to see that $x_{1} x_{2}$ is unique. For each point $z$ in $f\left(x_{1} x_{2}\right)$ the set $f^{-1}(z) \cap x_{1} x_{2}$ is convex, hence connected. Consequently, $f \mid x_{1} x_{2}$ is monotone, and $f\left(x_{1} x_{2}\right)$ is an arc from $y_{1}$ to $y_{2}$. The function $B: Y \times Y \rightarrow C(Y)$ given by $B\left(y_{1}, y_{2}\right)$ $=f\left(x_{1} x_{2}\right)$ is easily seen to be an arc-structure on $Y$.

TheOREM I-8-A. Let $f: X \rightarrow Y$ be a convex-monotone mapping, and let $B$ be the arc-structure on $Y$ induced by $f$. Then $f$ is $a \leqslant_{p}$-mapping for each $p$ in $X$ and $f(I(X)) \subseteq I(Y)$.

Proof. By the definition of the induced arc-structure $B$, the mapping $f \mid p x$ is monotone for each $p$ and $x$ in $X$. Thus $f$ is a $\leqslant_{p}$-mapping for each $p$ in $X$. Now $f(I(X)) \subseteq I(Y)$ by Theorem I-7-A.

I.9. The end set $E$. Following Lelek's definition for end point in the classical sense [25], we define the end set $E$ of $X$ to be $\{e \in X \mid$ if $e \in x y$, then $e=x$ or $e=y\}$.

Even in the special case when $X$ is a dendroid the end set $E$ can be very complicated (see [1], [24], and [25]). Our purpose here is to show that strong conclusions can be drawn when $(X, A)$ is arc-smooth and the end set $E$ is sufficiently well behaved.

The example in (I.3) shows that the end set is sometimes empty. However, whenever $(X, A)$ is arc-smooth, the end set is not empty as a consequence of the next lemma.

Lemma I-9-A. If $(X, A)$ is arc-smooth at $p$, then each arc $p x$ is contained in an arc pe with $e$ in the end set $E$.

Proof. Suppose there is no such point $e$. Then there is a sequence of points $\left\{y_{n}\right\}$ greater than $x$ such that $y_{n} \leqslant p y_{n+1}$ for each $n$ and such that no point $z$ satisfies $y_{n}<_{p} z$ for all $n$. Passing to subsequences if necessary, we can assume that $\left\{y_{n}\right\}$ converges to some point $y$. Since $<_{p}$ is closed by Theorem I-1-A, it follows that $y_{n} \leqslant_{p} y$ for each $n$, which is a contradiction.

THEOREM I-9-B. Let $X$ be a three-manifold with boundary a two-sphere $S^{2}$. If the end set $E$ coincides with $S^{2}$ and $(X, A)$ is arc-smooth at some point $p \in X \backslash E$, then $X$ is a three-cell.

Proof. Clearly $<_{p}$ is a closed partial order whose set of maximal elements coincides with $S^{2}$. Consequently $X$ is a three-cell by Theorem 3 of [37].

Our next result is a generalization of the theorem that if $P$ is the pseudo-arc, then the hyperspace $C(P)$ is a two-dimensional Cantor manifold [34]. Theorem II-1-A together with the observation that $C(P)$ has unique segments and that subcontinuum inclusion is a closed partial order show that $C(P)$ is arc-smooth at the point $P$. The same is true for any hereditarily indecomposable continuum. For an arbitrary continuum $Z$, the hyperspace $C(Z)$ need not be arc-smooth since $C(Z)$ need not be contractible. On the other hand, $C(Z)$ is sometimes homeomorphic to a cone in which case Theorem II-1-A shows that $C(Z)$ is arc-smooth. The reader is referred to [33] for these and related facts about hyperspaces. 
THEOREM I-9-C. Suppose that the continuum $X$ contains no separating points and that the end set $E$ is a continuum. If $(X, A)$ is arc-smooth at some point $p \in X \backslash E$, then no closed zero-dimensional set separates $X$.

Proof. First observe that $X$ is contractible with respect to the circle by Theorem I-6-A. Now according to Property 3 of [23] it suffices to show the following: (a) for each $e \in E$, pe $\cap E=\{e\}$, and (b) for each $z \neq p$ in $X \backslash E$ there are points $x$ and $y$ in $E$ such that $z \in x y$. Condition (a) is immediate from the definition of $E$. To prove (b), let $x$ be a point in $E$ such that $p z \subseteq p x$ (use Lemma I-9-A). By hypothesis, no point of $p z$ separates $X$. It follows easily that there is a point $w \in X \backslash p x$ such that $z \notin p z \cap p w$. Now let $y$ be a point of $E$ such that $p w \subseteq p y$. Thus $x y$ is a subarc of $p x \cup p y$ which contains $z$.

\section{Arc-smoothness for arbitrary continua.}

Convention. Throughout this section of the paper $X$ denotes an arbitrary continuum.

In the setting of arbitrary continua the following alternate definition of arcsmoothness (introduced in [12]) is of ten convenient.

Alternate Definition. The continuum $X$ is said to be arc-smooth at the point $p$ provided there exists a function $F: X \rightarrow C(X)$ such that for $x \neq p$ the set $F(x)$ is an arc from $p$ to $x$ and the following conditions are satisfied:

(a) $F(p)=\{p\}$,

(b) if $x \in F(y)$, then $F(x) \subseteq F(y)$, and

(c) $F$ is continuous.

To see that the two definitions are equivalent, we first suppose that $A$ is an arc-structure on $X$ such that $(X, A)$ is arc-smooth at $p$. Letting $F=A_{p}$ it is clear that $F$ satisfies the conditions of the alternate definition. Now suppose that $F$ : $X \rightarrow C(X)$ satisfies the alternate definition. For each $(x, y)$ in $X \times X$, let $A(x, y)$ denote the unique arc joining $x$ to $y$ in the subcontinuum $F(x) \cup F(y)$. Then $A$ : $X \times X \rightarrow C(X)$ is an arc-structure on $X$ and $A_{p}=F$. Consequently $A_{p}$ is continuous and $(X, A)$ is arc-smooth at $p$.

The alternate definition for arc-smoothness makes it apparent that cones over arbitrary compacta and star-like continua in $l_{2}$ are arc-smooth.

If $X$ is a convex continuum in $l_{2}$, then $X$ is clearly arc-smooth at each point in the sense that for each point $p$ in $X$ there is a function $F$ satisfying the conditions of the alternate definition. Observe that this is very different from requiring that $X$ admit a fixed arc-structure $A$ for which $(X, A)$ is arc-smooth at each point. The latter requirement forces $X$ to be a dendrite by Theorem I-2-E.

II.1. A partial order characterization. Purely order theoretic characterizations for smooth dendroids (i.e., metrizable generalized trees), smooth continua, and weakly smooth continua were obtained in [39], [26], and [28], respectively. In this section we present such a characterization for arc-smooth continua which is closely related to Theorems 2.6 and 2.8 of [9]. 
THEOREM II-1-A. The continuum $X$ is arc-smooth at the point $p$ if and only if $X$ admits a partial order $\leqslant$ such that

(a) $\leqslant$ is closed,

(b) $p$ is the zero of $\leqslant$, and

(c) for each $y \in X$ the lower set $L(y)=\{x \in X \mid x \leqslant y\}$ is an order arc.

Proof. Let $X$ be arc-smooth at $p$ and let $A$ be an arc-structure on $X$ such that $(X, A)$ is arc-smooth at $p$. Then the partial order $\leqslant_{p}$ defined in (I.1) is closed by Theorem I-1-A, and $\leqslant_{p}$ clearly satisfies (b) and (c).

Now suppose that $\leqslant$ is a partial order on $X$ satisfying (a), (b) and (c). Define $F$ : $X \rightarrow C(X)$ by the equation $F(x)=L(x)$. Clearly $F(p)=\{p\}$ and $F(x) \subseteq F(y)$ whenever $x \in F(y)$. The proof that $F$ is continuous is analogous to the proof that (b) implies (a) in Theorem I-1-A. Thus $X$ is arc-smooth at $p$ by the alternate definition.

II.2. Semigroups and semigroup actions. In their study of topological semigroups with zero and unit, Koch and McAuley [22] introduced a class of spaces called continua ruled by arcs. It is easy to see that the first four of the eight conditions in their definition are equivalent to our definition of arc-smooth continua. Continua satisfying their first four conditions were studied under the name ruled spaces by Eberhart [9] and under the name $K$-spaces by Stadtlander [36]. The reader is referred to [9] for various results concerning semigroup and semilattice structures on arc-smooth continua. In particular, it is worth noting that Corollary 2.3 of [9] shows that every continuum admitting a semilattice structure with identity is arc-smooth.

We next reinterpret the main theorem in [36] to obtain a useful characterization of arc-smooth continua in terms of thread actions. First we need some definitions.

By a thread $T$ we mean any topological semigroup (written multiplicatively) on the interval $[0,1]$ with 0 acting as a zero and 1 acting as a unit. We say that the thread $T$ acts naturally on the pointed continuum $(X, p)$ if there is a mapping $m$ : $T \times X \rightarrow X$ satisfying the following conditions for all $x$ in $X$ :

(a) $m(0, x)=p$,

(b) $m(1, x)=x$, and

(c) $m(s, m(t, x))=m(s t, x)$ for all $s, t \in T$.

Theorem II-2-A. Let $(X, p)$ be any pointed continuum, and let $T$ be any thread. Then $X$ is arc-smooth at $p$ if and only if $T$ acts naturally on $(X, p)$.

Proof. Stadtlander [36, p. 487] has observed that a $K$-space over a single point (called a $K$-space) satisfies the first four axioms for a continuum ruled by arcs. The result now follows immediately from our previous remarks and Theorem 1 of [36].

II.3. Contractibility of arc-smooth continua. Arc-smooth continua are contractible by Theorem I-6-A. Of course contractible continua need not be arc-smooth even in the class of dendroids. Our next result shows that a special kind of contractibility utilized by Isbell [17] in the study of injective metric spaces actually characterizes the arc-smooth continua. 
A free contraction [17] of a space $Z$ to a point $p$ is a homotopy $H: Z \times[0,1] \rightarrow$ $Z$ satisfying the following conditions for all $z$ in $Z$ :

(a) $H(z, 0)=p$,

(b) $H(z, 1)=z$, and

(c) $H(H(z, s), t)=H(z, \min \{s, t\})$ for all $s, t \in[0,1]$.

TheOREM II-3-A. The continuum $X$ is arc-smooth at $p$ if and only if $X$ is freely contractible to $p$.

Proof. Suppose that $H$ is a free contraction of $X$ to $p$. Let $T$ be the thread with $s t=\min \{s, t\}$ for $s, t \in[0,1]$. Define a mapping $m: T \times X \rightarrow X$ by $m(t, x)=$ $H(x, t)$. It follows that $T$ acts naturally on $(X, p)$. Hence $X$ is arc-smooth at $p$ by Theorem II-2-A.

The converse follows in a similar manner.

RemarK. It follows from Theorem II-3-A and Theorem 1.1 of [17] that every injectively metrizable continuum is arc-smooth at each of its points.

REMARK. A metric $d$ on the continuum $X$ is called strongly convex if for each pair of distinct points $x$ and $y$ in $X$ there is a unique arc from $x$ to $y$ which is isometric to the real line segment $[0, d(x, y)]$.

If $X$ admits such a metric, then it is easy to see that $X$ is arc-smooth at each point. The converse is an apparently difficult unsolved problem. By using ordinary multiplication in place of min multiplication in the proof of Theorem II-3-A, one sees that the converse is equivalent to a problem posed by Bing in [2]. Does $X$ admit a strongly convex metric provided that for each point $p$ in $X$ there exists a homotopy $H: X \times[0,1] \rightarrow X$ satisfying the following conditions for all $x$ in $X$ :

(a) $H(x, 0)=p$,

(b) $H(x, 1)=x$, and

(c) $H(H(x, s), t)=H(x, s t)$ for all $s, t$ in $[0,1]$ ?

THEOREM II-3-B. If $X$ is arc-smooth at $p$, then $X$ has a basis of closed contractible neighborhoods at $p$.

Proof. Let $A$ be an arc-structure on $X$ such that $(X, A)$ is arc-smooth at $p$, and let $d$ be a metric on $X$ which is radially convex at $p$ (see Theorem I-4-A). Then for each $\varepsilon>0$, the $\varepsilon$-neighborhood $N_{\varepsilon}(p)$ is clearly arc-smooth, hence contractible.

II.4. Some consequences of contractibility. In this section we collect a variety of results about arc-smooth continua which depend on their special contractibility properties.

THEOREM II-4-A. A finite dimensional continuum which is arc-smooth at each point is an absolute retract.

Proof. Such a continuum is contractible and locally contractible, hence an absolute retract by Corollary 10.5 of [4].

REMARK. Theorems I-2-E and II-4-A can be viewed as different ways of generalizing the fact that a dendroid which is smooth at each point is a dendrite (i.e., a one-dimensional absolute retract). 
THEOREM II-4-B. A one-dimensional continuum is arc-smooth if and only if it is a smooth dendroid.

Proof. By Theorem 1 of [7], every contractible one-dimensional continuum is a dendroid.

CoROllaRy II-4-C. A continuum is hereditarily arc-smooth (i.e., every subcontinuum is arc-smooth) if and only if it is a smooth dendroid.

Proof. Smooth dendroids are hereditarily smooth by Corollary 6 of [8]. Conversely, hereditarily arc-smooth continua are hereditarily arcwise connected, hence one-dimensional. Now apply Theorem II-4-B.

Our next theorem is a restatement of the main result in [38].

THEOREM II-4-D. A two-dimensional polyhedron is arc-smooth if and only if it is collapsible.

Proof. This follows directly from Theorem II-3-A and Theorem 1.8 of [17].

The continuum $X$ is called homogeneous if for each pair of points $x$ and $y$ in $X$ there exists a homeomorphism from $X$ onto $X$ taking $x$ to $y$. The Hilbert cube is a homogeneous arc-smooth continuum (see [11]).

THEOREM II-4-E. Every nondegenerate homogeneous arc-smooth continuum is infinite dimensional.

Proof. The proof in [17] of the analogous statement for injectively metrizable compacta depends on their free contractibility, and hence applies to arc-smooth continua as well.

The continuum $X$ is said to have the fixed point property if for each self-mapping $f$ there is a point $x$ in $X$ such that $f(x)=x$. Dendroids are known to have the fixed point property [3]; however, continua with arc-structures need not have the fixed point property even when arc-smooth since some cones over continua do not [20].

THEOREM II-4-F. If $X$ is an arc-smooth continuum which is uniquely arcwise connected or embeddable in the plane, then $X$ has the fixed point property.

Proof. Uniquely arcwise connected contractible continua have the fixed point property by Theorem 5 of [43]. Arcwise connected nonseparating plane continua have the fixed point property by Theorem 3 of [15].

THEOREM II-4-G. If the continuum $X$ is arc-smooth at the point $p$, then each closed set containing $p$ is the fixed point set of some self-mapping on $X$.

Proof. As in the remark on strongly convex metrics in (II.3), let $H: X \times[0,1]$ $\rightarrow X$ be a homotopy satisfying the following conditions for all $x$ in $X$ :

(a) $H(x, 0)=p$,

(b) $H(x, 1)=x$, and

(c) $H(H(x, s), t)=H(x, s t)$ for all $s, t$ in $[0,1]$. 
By Theorem 1 of [41] it suffices to prove that $H(x, t) \neq x$ for $x \neq p$ and $t \neq 1$. To this end, suppose that $t \neq 1$ and $H(x, t)=x$. Then $H(x, t)=H(H(x, t), t)=$ $H\left(x, t^{2}\right)$. Similarly, $H(x, t)=H\left(x, t^{n}\right)$ for all $n$. By continuity $H(x, t)=H(x, 0)$ and hence $x=p$ as desired.

COROLlARY II-4-H. If the continuum $X$ is arc-smooth at each point, then each of its closed sets is the fixed point set of some self-mapping on $X$.

\section{REFERENCES}

1. David P. Bellamy, An interesting plane dendroid, Fund. Math. 110 (1981), 37-54.

2. R. H. Bing, A convex metric with unique segments, Proc. Amer. Math. Soc. 4 (1953), 167-174.

3. K. Borsuk, $A$ theorem on fixed points, Bull. Acad. Polon. Sci. III 2 (1954), 17-20.

4. __ Theory of retracts, Polish Scientific Publishers, Warsaw, 1967.

5. J. H. Carruth, Topics in quasi-ordered spaces, Dissertation, Louisiana State Univ., Baton Rouge, La., 1966.

6. __ A note on partially ordered compacta, Pacific J. Math. 24 (1968), 229-231.

7. J. H. Case and R. E. Chamberlin, Characterizations of tree-like continua, Pacific J. Math. 10 (1960), 73-84.

8. J. J. Charatonik and Carl Eberhart, On smooth dendroids, Fund. Math. 67 (1970), 297-322.

9. Carl Eberhart, Some classes of continua related to clan structures, Dissertation, Louisiana State Univ., Baton Rouge, La., 1966.

10. __ A note on smooth fans, Colloq. Math. 20 (1969), 89-90.

11. M. K. Fort, Homogeneity of infinite products of manifolds with boundary, Pacific J. Math. 12 (1962), 879-884.

12. J. B. Fugate, G. R. Gordh, Jr. and Lewis Lum, On arc-smooth continua, Topology Proc. 2 (1977), 645-656.

13. G. R. Gordh, Jr., Concerning closed quasi-orders on hereditarily unicoherent continua, Fund. Math. 78 (1973), 61-73.

14. G. R. Gordh, Jr. and Lewis Lum, Radially convex mappings and smoothness in continua, Houston J. Math. 4 (1978), 335-342.

15. Charles L. Hagopian, A fixed point theorem for plane continua, Bull. Amer. Math. Soc. 77 (1971), 351-354.

16. J. G. Hocking and G. S. Young, Topology, Addison-Wesley, Reading, Mass., 1961.

17. J. R. Isbell, Six theorems about injective metric spaces, Comment. Math. Helv. 39 (1964), 65-76.

18. F. Burton Jones, Concerning non-aposyndetic continua, Amer. J. Math. 70 (1948), 403-413.

19. J. L. Kelley, Hyperspaces of a continuum, Trans. Amer. Math. Soc. 52 (1942), 22-36.

20. R. J. Knill, Cones, products and fixed points, Fund. Math. 60 (1967), 35-46.

21. R. J. Koch and I. S. Krule, Weak cutpoint ordering on hereditarily unicoherent continua, Proc. Amer. Math. Soc. 11 (1960), 679-681.

22. R. J. Koch and L. F. McAuley, Semigroups on continua ruled by arcs, Fund. Math. 56 (1964), 1-8.

23. J. Krasinkiewicz, No 0-dimensional set disconnects the hyperspace of a continuum, Bull. Acad. Polon. Sci. Sér. Sci. Math. Astronom. Phys. 19 (1971), 755-758.

24. J. Krasinkiewicz and P. Minc, Dendroids and their endpoints, Preprint No. 84, Institute of Mathematics, Polish Academy of Sciences, 1975.

25. A. Lelek, On plane dendroids and their end points in the classical sense, Fund. Math. 49 (1961), 301-319.

26. Lewis Lum, A quasi-order characterization of smooth continua, Pacific J. Math. 53 (1974), 495-500. 27. A characterization of local connectivity in dendroids, Studies in Topology, Academic Press,

New York, 1975, pp. 331-338.

28. __ Weakly smooth continua, Trans. Amer. Math. Soc. 214 (1975), 153-167.

29. __ Order preserving and monotone retracts of a dendroid, Topology Proc. 1 (1976), 57-61.

30. T. Mackowiak, Some characterizations of smooth continua, Fund. Math. 79 (1973), 173-186.

31. , On smooth continua, Fund. Math. 85 (1974), 79-95.

32. L. Mohler, A characterization of smoothness in dendroids, Fund. Math. 67 (1970), 369-376.

33. Sam B. Nadler, Jr., Hyperspaces of sets, Dekker. New York, 1978. 
34. T. Nishiura and C. J. Rhee, The hyperspace of a pseudoarc is a Cantor manifold, Proc. Amer. Math. Soc. 31 (1972), 550-556.

35. Raymond E. Smithson, $A$ note on acyclic continua, Colloq. Math. 19 (1968), 67-71.

36. David Stadtlander, Thread actions, Duke Math. J. 35 (1968), 483-490.

37. E. D. Tymchatyn, Some order theoretic characterizations of the 3-cell, Colloq. Math. 24 (1972), 195-203.

38. __ Partial order and collapsibility of 2-complexes, Fund. Math. 77 (1972), 5-7.

39. L. E. Ward, Jr., Mobs, trees, and fixed points, Proc. Amer. Math. Soc. 8 (1957), 798-804.

40. __ Rigid selections and smooth dendroids, Bull. Acad. Polon. Sci. Sér. Sci. Math. Astronom. Phys. 19 (1971), 1041-1044.

41. _ Fixed point sets, Pacific J. Math. 47 (1973), 553-565.

42. G. T. Whyburn, Analytic topology, Amer. Math. Soc. Colloq. Publ., vol. 28, Amer. Math. Soc., Providence, R. I., 1942.

43. G. S. Young, Fixed-point theorems for arc-wise connected continua, Proc. Amer. Math. Soc. 11 (1960), 880-884.

Department of Mathematics, University of Kentucky, LeXINGTON, Kentucky 40506

Department of Mathematics, Guilford College, Greensboro, North Carolina 27410

Department of Mathematics, Salem College, Winston-Salem, North Carolina 27108 